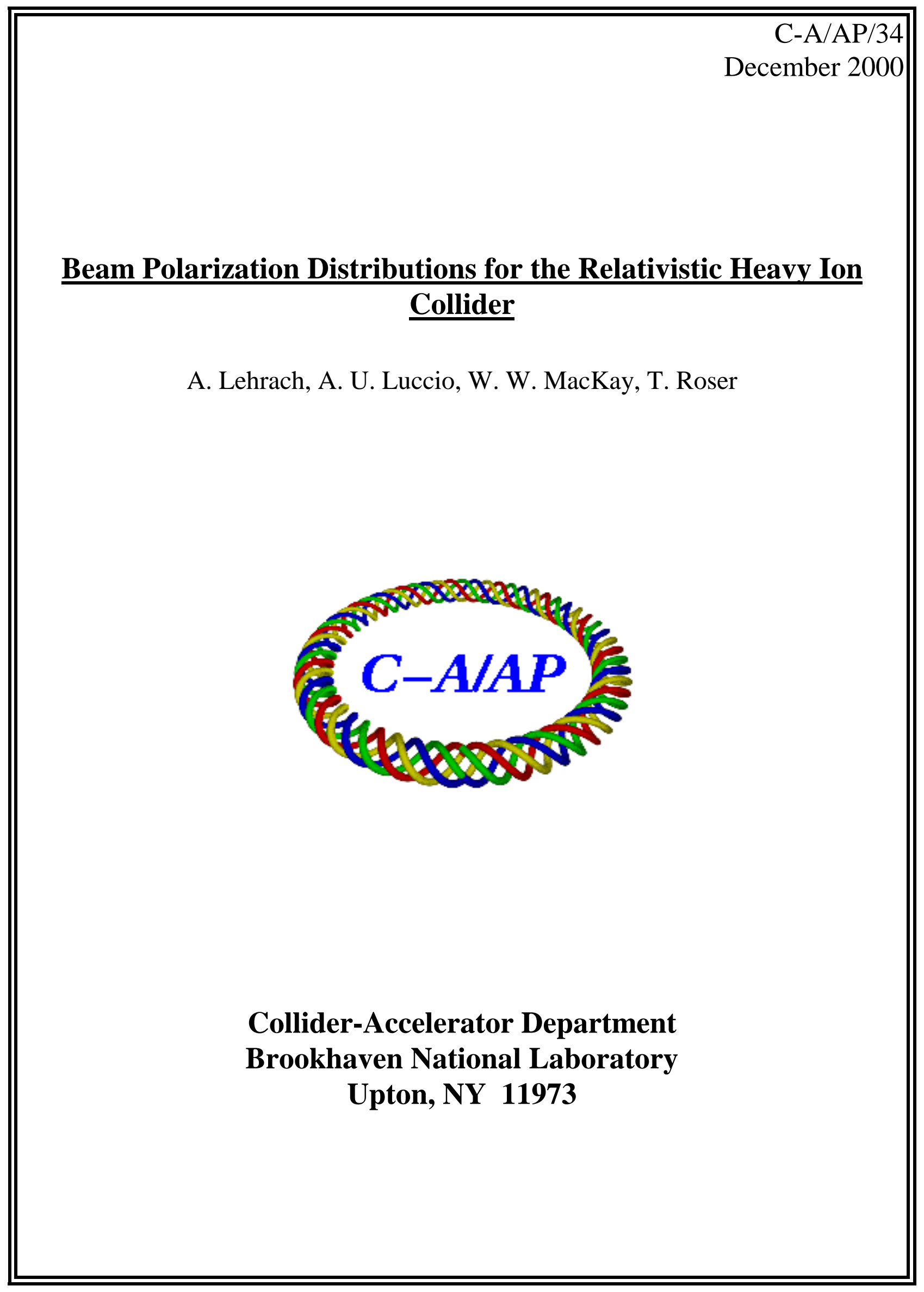




\title{
Beam Polarization Distributions for the Relativistic Heavy Ion Collider *
}

\author{
A. Lehrach, A.U. Luccio, W.W. MacKay, T. Roser \\ Brookhaven National Laboratory, Upton, NY 11973, USA
}

\begin{abstract}
In a high energy accelerator the polarization distribution of the beam can vary substantially across the beam. This decreases the amount of polarization provided to experiments. A method has been developed to calculate the beam polarization distribution based on adiabatic particle and spin excitation by inducing coherent betatron oscillations using an ac dipole. In earlier studies we calculated the beam polarization distribution in Brookhaven's Relativistic Heavy Ion Collider (RHIC) at fixed energy to compare spin motion on and away from strong spin resonances [1]. In this paper we present calculations of the beam polarization distribution during spin resonances crossing in RHIC.
\end{abstract}

\section{INTRODUCTION}

In order to investigate the polarization distribution of the beam in a circular accelerator, the invariant spin field has to be calculated. The invariant spin field $\vec{n}(\vec{z})[2]$ depends on the position $\vec{z}$ of the particle in the six dimensional phase space. A particle with the initial spin $\vec{s}_{i}$ at the phase space position $\vec{z}_{i}$ has the final spin $\vec{s}_{f}$ after it has been transported to the phase space point $\vec{z}_{f}$ during one turn in a storage ring. If $T_{t=1}$ is the one turn spin transfer matrix, then for every phase space point $\vec{z}_{i}$ a spin field vector $\vec{n}\left(\vec{z}_{i}\right)$ exists such that

$$
\vec{n}\left(\vec{z}_{f}\right)=T_{t=1} \vec{n}\left(\vec{z}_{i}\right) .
$$

The spin follows the invariant spin field if the motion of the spin is adiabatic. A particle spin vector with an initial angle $\phi$ with respect to the corresponding invariant spin field vector $\vec{n}\left(\vec{z}_{i}\right)$ will be rotated around $\vec{n}\left(\vec{z}_{i}\right)$ every time it comes close to $\vec{z}_{i}$. If the spin motion is adiabatic, the angle $\phi$ is constant. The time averaged polarization at $\vec{z}_{i}$ will be parallel to $\vec{n}\left(\vec{z}_{i}\right)$ with a magnitude of one if the spin vectors are parallel to the invariant spin field. The maximum time averaged polarization $P_{l i m}$ of the beam is given by the average of the invariant spin field vectors $\langle\vec{n}\rangle$ over the beam, and therefore depends on the phase space distribution

*) Work performed under the auspices of the U.S. Department of Energy. 
of the beam [3]. A large angle spread of $\vec{n}(\vec{z})$ leads to low maximum time averaged polarization. Thus the invariant spin field has to be calculated to determine the amount of maximum beam polarization delivered to experiments. A method to calculate the invariant spin field is stroboscopic averaging [4], which is based on multi-turn tracking and averaging of the spin viewed stroboscopically from turn to turn at one position in the ring. The invariant spin field has also been studied using a method called adiabatic anti-damping [3], which is very similar to the method presented here. In our study, the motion of the particle and spin is adiabatically excited with coherent betatron oscillations using an ac dipole [1]. Spin and particle motion were calculated using the spin tracking program SPINK [5].

\section{CALCULATION OF THE INVARIANT SPIN FIELD}

A controlled betatron oscillation is introduced by an ac dipole to calculate the invariant spin field. The ac dipole is slowly energized to the desired field and back to zero field in a way that the resulting orbit and spin motion are adiabatic. Adiabaticity is maintained during this process if the spin and particle return to their initial positions after de-energizing this device. In order to get a large coherent amplitude $Z_{c o h}$, the modulation tune of the ac dipole, defined as the oscillation frequency of the ac dipole field divided by the revolution frequency of the particles in the accelerator, has to be close to the fractional betatron tune [6]:

$$
Z_{c o h}=\frac{1}{2} \beta_{z} \frac{B_{a c} l}{B \rho} \frac{1}{2 \pi \delta} .
$$

In Eq. $2, \beta_{z}$ is the betatron amplitude at the location of the ac dipole, $\delta$ the difference between the modulation tune of the ac dipole field and the fractional betatron tune, $l$ the length of the ac dipole, $B_{a c}$ the amplitude of the ac dipole field, and $(B \rho)$ the magnetic rigidity of the beam. If the spin of the particle on the closed orbit is started parallel to the corresponding spin field vector ${ }^{\dagger}$, and the ac dipole excites the motion of the spin adiabatically, then the spin of the particle remains parallel to the invariant spin field during the excitation. In this case one particle spin tracking is sufficient to calculate the invariant spin field for the entire phase space, as shown in the next section.

\section{INVARIANT SPIN FIELD DURING ACCELERATION}

In the presented simulation, the design RHIC lattice with a vertical tune of $\nu_{y}=29.18$ was chosen without field errors and misalignments of the magnets [7]. The motion of the invariant spin field has been investigated during crossing the strongest intrinsic resonance in the energy range of RHIC. The amplitude of the ac dipole field was increased in 5000 turns starting at $\gamma G=416$, kept constant at the

†) The $\vec{n}$ axis on the closed orbit is calculated using stroboscopic averaging [4] 
desired field strength during spin resonance crossing for 13600 turns, and decreased back to zero amplitude in 5000 turns just before reaching $\gamma G=430$. The particle and spin return adiabatically to their initial position. To determine the maximum beam polarization that can be provided to experiments, the invariant spin field is investigated at the interaction point of the PHENIX experiment.
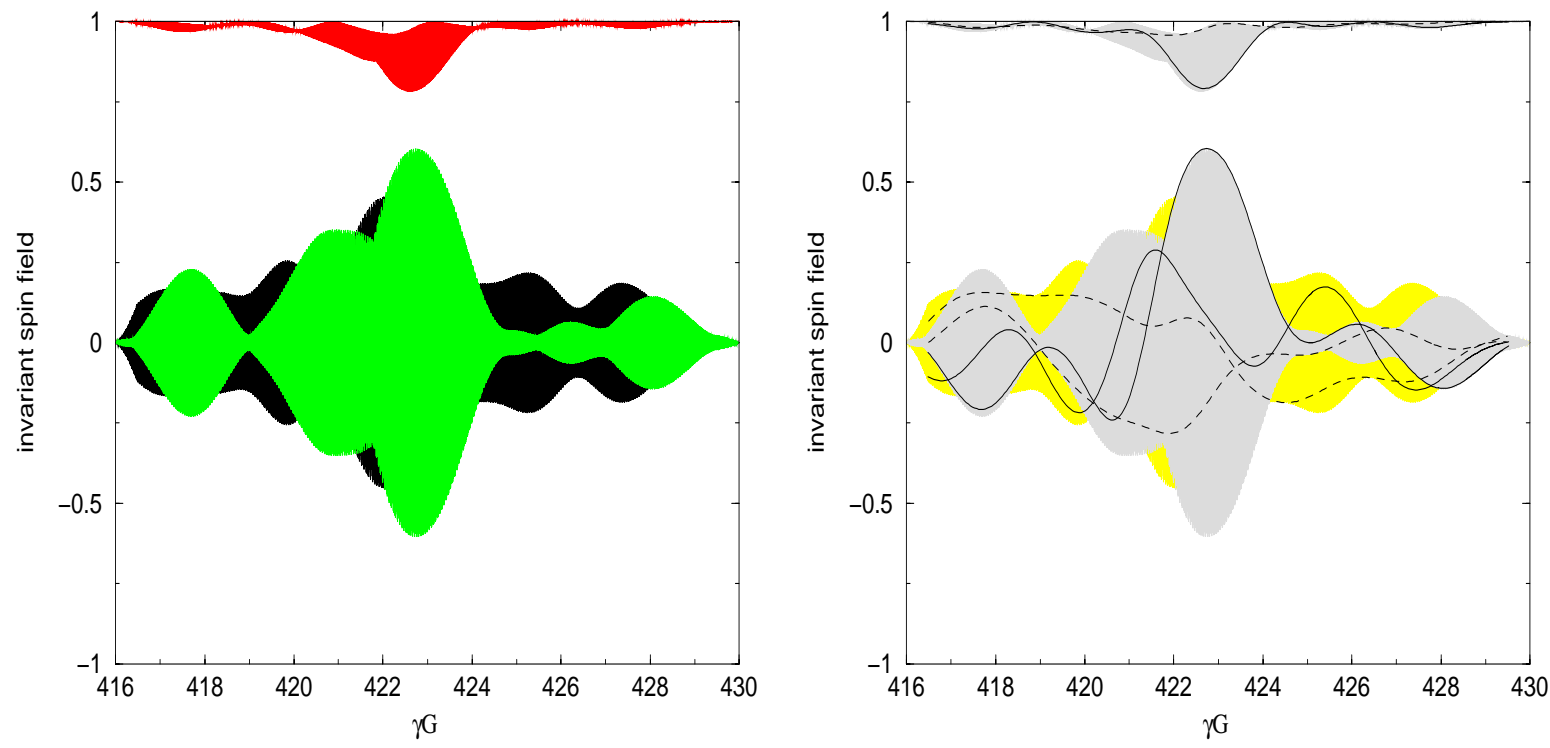

FIGURE 1. Components of the invariant spin field versus energy in units of $\gamma G$. The left plot shows the invariant spin field for a particle on a phase space ellipse with a normalized vertical emittance of $5 \pi \mathrm{mm}$ mrad. The upper curve is the vertical component of the invariant spin field, the two lower curves are the horizontal and longitudinal components. The lines in the plot on the right side show the invariant spin field for two selected vertical phase space points with maximum orbit (solid line) and angular displacement (dashed line).

Fig. 1 shows, that spin resonances in this energy range are overlapping. The invariant spin field is strongly depending on the position in vertical phase space. For different phase space points the motion of the invariant spin field is quite different. In this energy range the maximum time averaged polarization of the beam with a normalized vertical emittance of $5 \pi \mathrm{mm}$ mrad will be higher than 0.75 for any vertical phase space distribution. Fig. 2 shows the dependence of the invariant spin field on the vertical emittance of the beam. As expected, the excitation of the invariant spin field is increasing with vertical emittance; whereas the width (full width half maximum) of the invariant spin field distribution at spin resonances is constant. Up to a vertical excitation of $20 \pi \mathrm{mm}$ mrad the spin motion stays adiabatic, even during crossing the strongest intrinsic resonance when the oscillation of the invariant spin field is very large with a vertical component continuously flipping sign. The beam energy to deliver a polarized beam to experiments has to be chosen carefully in this energy range. Even for a beam with a normalized vertical emittance of $20 \pi \mathrm{mm}$ mrad it is possible to get a maximum time averaged polariza- 


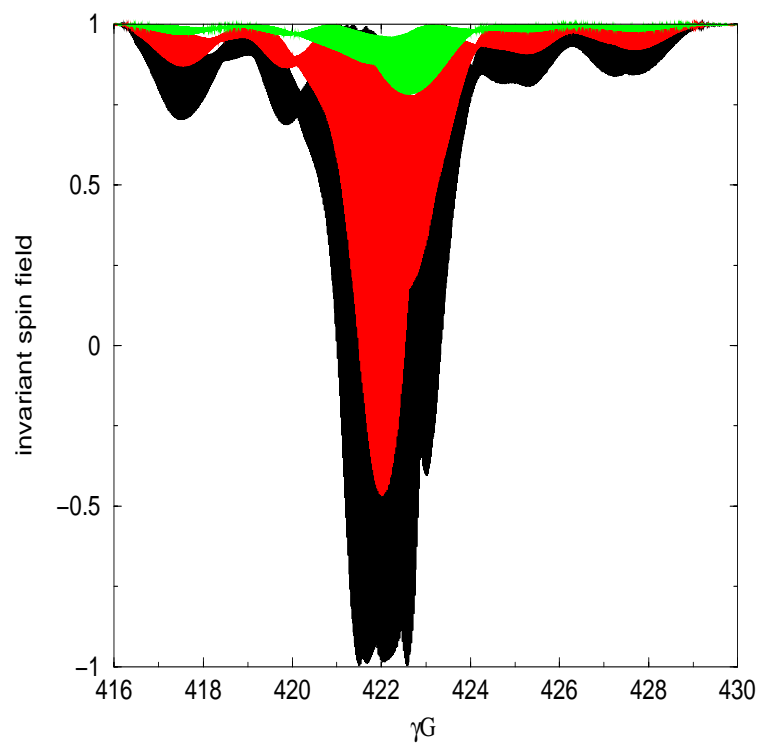

FIGURE 2. Vertical component of the invariant spin field versus energy in units of $\gamma G$ for particles on different phase space ellipse corresponding to a normalized vertical emittance of $5 \pi \mathrm{mm} \mathrm{mrad}$ (upper curve), $10 \pi \mathrm{mm} \mathrm{mrad}$ (curve in the middle), and $20 \pi \mathrm{mm}$ mrad (lower curve).

tion higher than 0.9 , if polarized beam is provided to experiments away from spin resonances, at energies around $\gamma G=418.5$ or 426.5 .

\section{POLARIZATION LOSSES DURING SPIN RESONANCE CROSSING}

Polarization losses occur if the spin vector is not able to follow the motion of the invariant spin field adiabatically during spin resonance crossing. Thus the angle $\phi$ between the spin vector and its corresponding invariant spin field vector is increasing. In this calculation the coherent amplitude of the betatron oscillation was increased to observe non-adiabatic spin motion. Since the invariant spin field is vertical after turning off the ac dipole, the maximum time averaged polarization equals one. Therefore polarization losses for different cases can be investigated by comparing the vertical component of the spin vector at $\gamma G=430$. Fig. 3 shows the non-adiabatic spin motion for different vertical particle excitations. The spin vector starts to oscillate around the corresponding invariant spin field vector during crossing of the strongest intrinsic resonance, and remains non-parallel to the invariant spin field after turning off the ac dipole. As can be seen, the polarization losses are increasing with beam excitation and even spin flips are possible. 


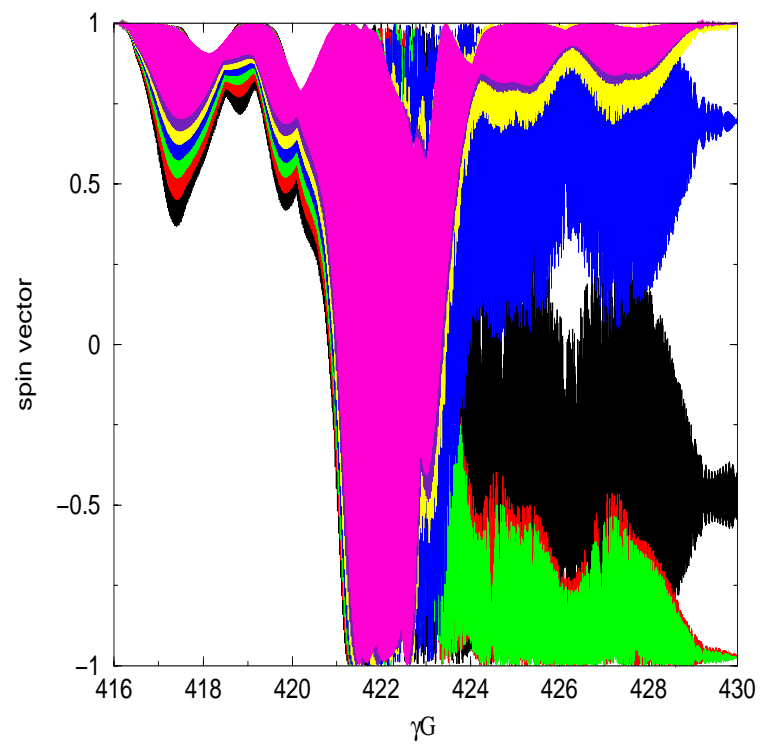

FIGURE 3. Vertical component of the spin vector versus energy in units of $\gamma G$ for particles on equally spaced phase space ellipse of normalized vertical emittances between 20 and $40 \pi \mathrm{mm}$ mrad.

\section{CONCLUSION AND OUTLOOK}

Polarization distributions for RHIC have been calculated by exciting the motion of particle and spin with an ac dipole field. It has been shown that the maximum time averaged polarization is depending on the vertical phase space distribution of the beam and strongly decreasing with the vertical beam size. The simulation indicates that some energy ranges in the vicinity of spin resonances should be excluded from being used for experiments at RHIC. Furthermore polarization losses during spin resonance crossing have been investigated.

This studies will be continued to learn more about beam polarization distributions and spin resonance crossing in RHIC. In addition spin tunes will be calculated by analyzing spin oscillation of particles with non-parallel initial spin direction to the corresponding invariant spin field vector.

\section{REFERENCES}

1. A. Lehrach et al., DESY-Proc-1999-03, 210 (1999).

2. Ya. Derbenev, A.M. Kondratenko, Sov. Phys. JETP 37(6), 968 (1973).

3. M. Vogt et al., Proc. of EPAC 98, Stockholm, 1362 (1998).

4. G.H. Hoffstätter et al., Phys. Rev. E 54, 4240 (1996).

5. A.U. Luccio et al., Proc. of PAC 99, New York, 1578 (1999).

6. M. Bai et al., Phys. Rev. E 56, 6002 (1997).

7. Design Manual - Polarized Proton Collider at RHIC, BNL (1998); http://www.agsrhichome.bnl.gov/RHIC/Spin/. 